CHRONIC KIDNEY DISEASE

\section{suPAR in CKD}

Soluble urokinase-type plasminogen activator receptor (suPAR) has been implicated in the pathogenesis of kidney disease. A new report now describes associations between elevated plasma suPAR levels, decline in renal function and incident chronic kidney disease (CKD) in individuals with normal kidney function at baseline.

To assess the utility of suPAR as a potential biomarker for new-onset CKD, Jochen Reiser and colleagues investigated the relationship between baseline suPAR levels and decline in estimated glomerular filtration rate (eGFR) in 2,292 individuals enrolled in the Emory Cardiovascular Biobank for whom follow-up eGFR measurements were available. "suPAR levels were independently predictive of eGFR decline in patients with a wide range of baseline kidney function-down to an eGFR of $45 \mathrm{ml} / \mathrm{min} / 1.73 \mathrm{~m}^{2}$," says Reiser. Over a median follow-up of 3.7 years, the annual change in eGFR was $-0.9 \mathrm{ml} / \mathrm{min} / 1.73 \mathrm{~m}^{2}$ among participants in the lowest quartile of suPAR levels and $-4.2 \mathrm{ml} / \mathrm{min} / 1.73 \mathrm{~m}^{2}$ among participants in the highest quartile $(P<0.001)$.

Among 1,335 participants who had a baseline eGFR $\geq 60 \mathrm{ml} / \mathrm{min} / 1.73 \mathrm{~m}^{2}$, higher baseline suPAR level was also associated with a significantly greater incidence of CKD: participants in the highest suPAR quartile had a threefold greater risk of developing CKD than participants in the lowest quartile.

Reiser is planning further studies to investigate suPAR in various other cohorts, define its possible interaction with APOL1 and clarify the contribution of different suPAR forms to various renal diseases. "The hypothesis is that suPAR behaves in a similar manner to cholesterol, whereby plasma levels as well as subforms matter," he explains. "We will also try to perform intervention studies to see if lowering suPAR levels changes renal outcomes."

Susan J. Allison

ORIGINAL ARTICLE Hayek, S. S. et al. Soluble urokinase receptor and chronic kidney disease. N.Engl.J.Med. 373, 1916-1925 (2015) 